\title{
Priorities in biomedical research
}

\section{Indices of burden}

\author{
D. A. K. BLACK 1 AND J. D. POLE 2 \\ University of Manchester ${ }^{1}$ and Department of Health and Social Security 1.2
}

\begin{abstract}
Black, D. A. K. and Pole, J. D. (1975). British Journal of Preventive and Social Medicine, 29, 222-227. Priorities in biomedical research: indices of burden. The relative burden of different diseases is a legitimate component in the consideration of priorities for biomedical research. Simple prevalence statistics do not directly reflect the different burdens on services imposed by diseases of comparable prevalence. This paper sets out for each of 54 categories of disease five indices of the burden on services, based respectively on inpatient days, outpatient referrals, consultations in family practice, sickness benefit, and loss of expectation of life. There is considerable variation in the rank-order of categories of disease, in their contribution to the five burdens; but for each burden the number of categories accounting for $50 \%$ of the total burden is not large, ranging from 3 to 9 out of the possible 54 .
\end{abstract}

The definition of priorities in research is commonly arrived at by the consensus judgement of a committee, whose members consider implicitly or explicitly the importance of a field of research; the extent to which effort is already being deployed; and the scientific feasibility and interest of what is proposed. The third of these considerations can scarcely be quantified, except by a rough estimate of the odds of success or failure; and the second is likewise very largely a judgement of quality rather than of quantity. There are certainly also difficulties in estimating the first component: the 'importance' of a field of research; but in this paper we propose a quantitative assessment of the relative burden imposed on the health service and on the health of the nation by a number of broad categories of illness.

The disease categories were derived by aggregation of the much more numerous diseases listed in the International Classification of Diseases to yield the manageable figure of 54 categories. The categories are virtually comprehensive of established disease states, but they do not include a number of 'residual' causes of burden, listed at the foot of the table, of which the most important are 'certain causes of perinatal morbidity', 'symptoms and ill-defined conditions', and 'prophylactic procedures and medical examinations'. The categories used are virtually exclusive, with minor exceptions where diseases of particular importance-such as, breast cancer, multiple sclerosis, and epilepsy-are itemized separately, in addition to being included in a mor general category.

We have used five indices of burden, the first thro being related to use of resources, the remaining two being related to morbidity and mortality:

\section{InPatient Days}

Derived mainly from Hospital In-Patient Enquiry (HIPE) Part I 1972, Table 9. Data on Psychiatry, $\overrightarrow{\vec{\partial}}$ Preconvalescent, Convalescent, Private and Staff Beds derived from Form SH3 returns.

\section{Outpatient Referrals}

Office of Population Censuses and Surveys (OPCS) Morbidity Statistics from General Practice. Second National Study 1970-71, Table 18.

3. GP Consultations Surgery consultations, OPCS Morbidity Statistics, $\bigcirc$ Table 10.

Home visits, OPCS Morbidity Statistics, Table 20. The home visits have been weighted by a factor of $N$ 4 , and added to the surgery consultation rates to give a notional overall rate per 1000 population.

\section{MORBIDITY}

Social Security Statistics, 1973. Tables 3.82 ando 3.83. Sickness and/or invalidity benefit. These figures 
refer to insured persons only, so that they give an accurate picture of the cost in sickness benefit of the disease categories, but not of total morbidity.

\section{MoRtality}

Registrar General's Statistical Review 1972. Table 19 and Appendix B-abridged life table.

An analysis of the tables has been made to differentiate between the major causes of resource use, morbidity, and mortality. While these are significantly different for each of the five indices used, more than half of the burden in each case is accounted for by comparatively few disease categories. This is seen at its most extreme in bed occupancy; mental illness and mental handicap alone account for about half the beds. Even in the most diverse group, that of GP consultations, half of the burden is accounted for by only nine categories of disease, and only 15 categories are found each to account for $2 \%$ or more of the total burden. The unavoidable use of rather broad categories clearly contributes to this effect-that is, the number of major causes of each type of burden is quite small; but this artefact does not account for the great discrepancy between the major causes of the different burdens considered.

For each of the five indices the major causes of burden in rank order are listed in Appendix I, and the conditions which account for over $50 \%$ of the burden are indicated. At the end of each list the percentage of the total burden accounted for by all the conditions listed is given, and the percentage of the burden not accounted for by the 54 disease categories used. The tabulation on which the analysis has been based is shown in Appendix II. In general, the categories in the two appendices are the same, but in some instances-for example, digestive diseases-the original categories have been pooled, when excessive subdivision in the list of 54 disease conditions seemed to be introducing bias.

Comment on lists of this type is clearly an arbitrary matter, but some points may be worth making on the individual lists, and in general.

\section{COMMENT ON INDIVIDUAL LISTS}

\section{INPATIENT DAYS}

The outstanding feature, already well-recognized, is the preponderant place of mental illness and mental handicap in hospital bed-occupancy. This preponderance would be less in terms of resourcecost, either in finance or in manpower, and this would also apply to the third major cause of bed-occupancy, convalescence from cerebrovascular episodes. The proportion of 'high-cost' or 'acute' beds in the case of the other eight conditions listed would be substantially higher. The omission of respiratory diseases from the ranking is something of an artefact, as if summated they would aggregate $3 \cdot 80 \%$, distributed rather evenly over the categories 'respiratory infection', 'bronchitis and asthma', and 'other respiratory disease'.

Although 'bed-occupancy' is a better index of the use of hospital resources than is 'discharges + deaths', it too contains bias, in that 'short-stay' conditions, such as attempted suicide, impose a more intensive burden in assessment and care than does chronic illness, which accounts for a higher proportion of bed-occupancy.

\section{OUTPATIENT REFERRALS}

Largely in consequence of the relatively smaller burden imposed by mental illness, the outpatient work of the hospital sector is more varied than the inpatient work. This is crudely indicated by the $50 \%$ load' being made up of eight conditions in place of only three for the inpatient load, and it applies also to GP consultations.

If all bone and joint conditions were summated, they would top the list. Similarly, if respiratory disorders were summated, they would come fourth in the list. Artefacts of this type indicate that these lists are not appropriate for drawing fine conclusions, only for a 'broad-brush approach'.

Attention may be drawn to skin disorders, which account for only $0.76 \%$ of bed-occupancy, but come high in both outpatient referrals and GP consultations. Moreover, the figures given for 'skin diseases' are an underestimate, since bacterial and viral infections of the skin are categorized as 'infections', not as 'skin diseases'.

\section{GP Consultations}

Comparison of this list with the previous one (outpatient referrals) gives some information on the pattern of hospital referrals. For example-a relatively small proportion of GPs' work load in relation to respiratory disorders is passed on by reference to hospital; whereas the $2 \%$ of GP consultations due to neurological disorders accounts for $9.82 \%$ of the patients whom they refer to hospital outpatients.

\section{Days on Sickness Benefit}

This is a better index of 'cost' to the community than it is of morbidity, since it omits the nonemployed and non-insured population, who also suffer morbidity, and indeed account for a large part of it. Respiratory disorders account for more than one-fifth of this burden. 


\section{'MORTALITY'}

In spite of the weighting against them arising from the use of 'loss of life expectancy', the major causes of mortality are still those which operate predominantly in the later decades of life. A notable exception to this is the appearance of 'accidents and suicides' relatively high in the list.

\section{General Comment}

There are several reasons why only very broad indications of priority can be drawn from this material. One such reason is the imperfection of both the data and the analysis. Another lies in the different types of burden which have been evaluated; emphasis on the hospital burden alone would be different on the two different criteria of bedoccupancy and outpatient work; and the burden on primary care is different again. Moreover, the indices based on use of resources would suggest priorities different from those based on 'need', as reflected in morbidity and mortality. Both the possibility and the propriety of making a valuejudgement between resource-saving, the avoidance of suffering, and the prolongation of life are open to question. Within each of the indices used, there is an implied objective which is worth pursuing; but there is no critique for ranking these objectives in order. A further point of substance is that there are conditions which cause much suffering, but which account for only a very small part of resource-usage, morbidity, and mortality. For example-the highest percentage of any of the indices of burden accounted for by eye diseases is only $1.28 \%$ (GP consultations); ear diseases, including deafness, reach their highest percentage of only $2.60 \%$ on the same index. Yet these are conditions of undeniable importance.

Two further, rather more theoretical, incentives to caution in using this analysis may be mentioned. The data available inevitably reflect what is being done with existing resources, and what is happening to people; we cannot however assume that 'whatever is, is right'. The second point, a familiar one, is that a clear service need for research cannot be equated with the likelihood of that research being profitable; the problems involved may be clearly insoluble, there may be a lack of skilled workers, there may be stultification of research by either a lack or a superfluity of suitable patients.

These are real difficulties, but they should not be made an excuse for abandoning the attempt to define priorities for biomedical research, derived from service needs. For these reasons, the priorities so defined are not the only priorities to be considered in directing research; and even within their own framework, they can only be relative, and not $\omega$ absolute. In other and simpler words, there can be $\varnothing$ no health problem which carries no priority.

If a priority list is to be useful, it should be ?? reasonably simple and manageable. We have $\vec{F}$ therefore considered only those categories which fall $\stackrel{\text { ? }}{+}$ in the list of conditions which together cause at least $50 \%$ of the burden represented by at least one index; and after each category, we have indicated the index, $\stackrel{\vec{\phi}}{\vec{\sigma}}$

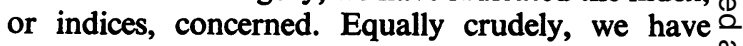
averaged the burden relating to each condition under $\%$ all five indices, to derive a rank order. Since there $\overrightarrow{0}$ are three indices related to resource use, and only two related respectively to morbidity and mortality, $\vec{\omega}$ this introduces some bias, but probably no greater than any other way of handling the data.

\begin{tabular}{|c|c|c|c|}
\hline Category & & $\begin{array}{l}\text { Indices Mainly } \\
\text { Affected }\end{array}$ & $\begin{array}{l}\text { Percentage of } \\
\text { Total Burden }\end{array}$ \\
\hline 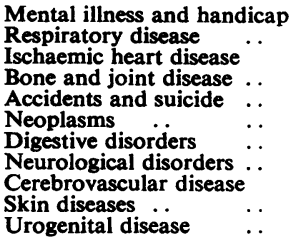 & $\begin{array}{l}\cdots \\
\cdots \\
\cdots \\
\cdots \\
\cdots \\
\cdots \\
\cdots \\
\cdots\end{array}$ & $\begin{array}{l}1,2,3,4 \\
3,4 \\
4,5 \\
2,3,4 \\
2,3,4 \\
5,3,4 \\
2,5 \\
1,3 \\
2,3\end{array}$ & \begin{tabular}{rl}
$13 \cdot 60$ & \\
$13 \cdot 47$ & 7 \\
$6 \cdot 59$ & 0 \\
$6 \cdot 38$ & \multicolumn{1}{c}{} \\
$6 \cdot 25$ & $\overparen{1}$ \\
$6 \cdot 08$ & \multicolumn{1}{c}{} \\
$4 \cdot 56$ & 0 \\
$4 \cdot 11$ & \\
$3 \cdot 74$ & 0 \\
$2 \cdot 55$ & \\
$2 \cdot 31$ &
\end{tabular} \\
\hline & & & $69.64=$ \\
\hline
\end{tabular}

* Where more than one index number is given for a category, the number corresponding to the most severely affected index is in italics. The indices are numbered as in Appendix I, i.e.:

1. Inpatient days

2. Outpatient referrals

3. General-practitioner consultations

4. Days of sickness-benefit

5. Mortality, as loss of life-expectancy

To anyone familiar with the diversity of clinicalo practice this list may seem a rather cumbrous way of quantitating the obvious, but there is at least one way in which it fails to do so. By the artefact of separating ischaemic heart disease and cerebro- 3 . vascular disease, not to mention other disorders of blood vessels, it seriously underestimates the role of $₹$ vascular degeneration in causing morbidity and $\mathrm{O}$ death. It may also be noted, however, that the conditions in this list account collectively for $70 \%$ of the total burden, on the five indices used.

Requests for reprints: Sir Douglas Black, Chief Scientist, Department of Health and Social Security, Alexander Fleming House, Elephant and Castle, London SE1 6BY.

We are grateful to Mrs B. D. Latter for statistical assistance. Views expressed or implied in the paper ared those of the authors. 


\section{APPENDIX I}

RANK ORDER OF CATEGORIES OF DISEASE, ACCORDING TO FIVE TYPES OF BURDEN

For each list, a double line is drawn below the conditions which cause $50 \%$ of the total burden. Under each list is given the percentage of total burden accounted for by the conditions listed; and the percentage of total burden due to causes other than classifiable disease, described as 'unaccounted burden'.

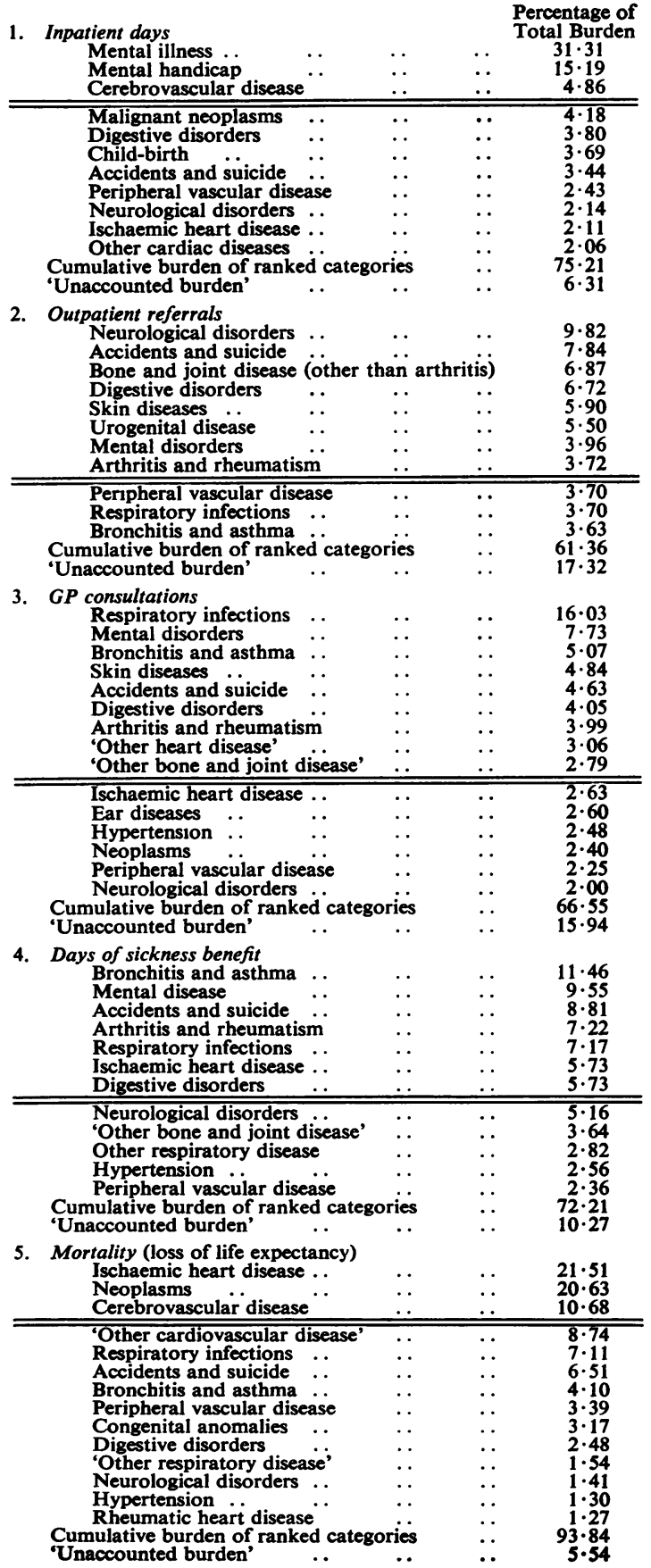


APPENDIXOII

DISBENEFITS ATTRIBUTABLE TO IRि.

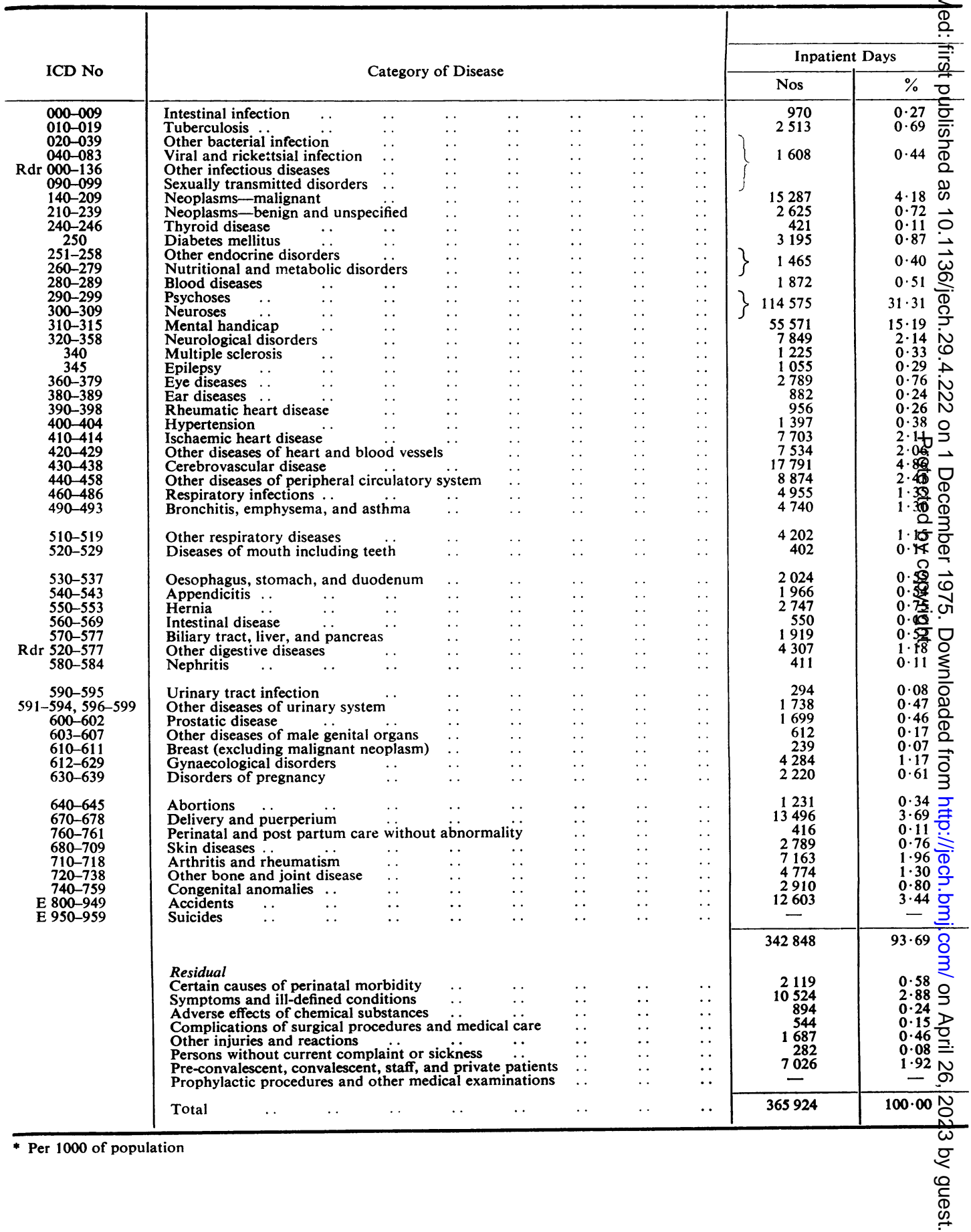


HEALTH BY DIAGNOSTIC CATEGORY

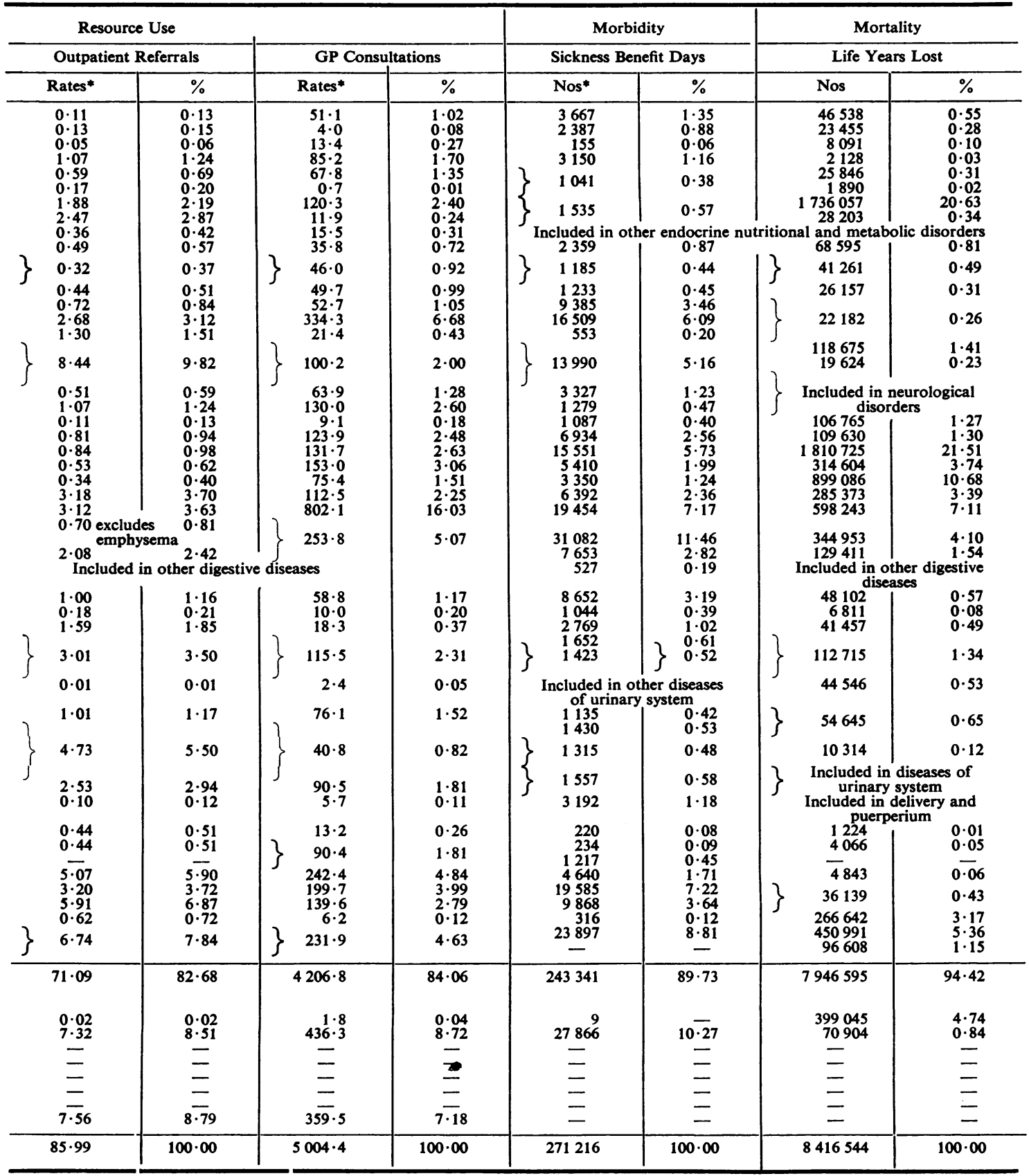

